\title{
The COVID-19 Pandemic: The Role of Body Exercise
}

\author{
Faisal Muhammad (iD) ${ }^{1, *}$ \\ ${ }^{1}$ Department of Public Health, Faculty of Allied Health Sciences, Daffodil International University, Dhaka, Bangladesh \\ "Corresponding author: Department of Public Health, Faculty of Allied Health Sciences, Daffodil International University, Mirpur Road, Dhaka, Bangladesh. Tel: \\ +880-1723406483, Email: fokkanya@yahoo.com \\ Received 2020 June 02; Accepted 2020 June 16.
}

Keywords: COVID-19, Pandemic, Exercise, Physical Health

\section{Dear Editor,}

As the new novel coronavirus disease (COVID-19) continues to spread across the globe, people are being requested to stay at home (self-quarantine). Staying at home inactive for a long period is a big challenge to our health. Currently, in most countries, fitness centers and other places where people used to do exercises are temporarily closed. The low level of physical activity and sedentary behavior may have directly or indirectly negative effects on our health, well-being, and quality of life. The World Health Organization (WHO) recommends moderate-intensity (for at least 150 minutes) or vigorous-intensity (for at least 75 minutes) physical activity per week. Physical activity can be a valuable tool to continue to protect health during the COVID-19 crisis (1). The research has discovered that exercise can reduce the risk of some respiratory problems such as acute respiratory distress syndrome (ARDS). The ARDS is a respiratory failure characterized by the rapid onset of widespread inflammation in the lungs that prevents oxygen from reaching the organs, and it has been reported in many COVID-19 patients (2). The Centre for Disease Control and Prevention (CDC) reported that the majority (around $42 \%$ ) of the COVID-19 hospitalized patients develop ARDS and the ARDS was the cause of death among many patients infected with SARS-CoV-2 (3).

During the COVID-19 pandemic, staying physically healthy or active can improve both emotional and mental health, and all these can boost the immune system. For the exercise of our body, we do not need too much space; sometimes, only simple exercises such as jumping, marching, yoga, and simple body stretches benefit the body and improve mental health (4). Moderate and vigorous physical activity mobilizes billions of immune cells, particularly a type of cells that can recognize and kill viral-infected cells. Exercise can help in maintaining the immunity of an individual by releasing various proteins, particularly muscle-derived cytokines (like IL-6, IL-7, and IL-15). Elderly people comprise a vulnerable group during the COVID19 crisis, and exercise can help them to be less susceptible to this infection. Currently, there are no scientifically proven effects of exercise on coronaviruses. However, several studies have revealed that exercise can protect an individual from many viral infections, such as rhinovirus, herpes viruses, influenza viruses, and etc. (5-7). Regular or moderate-intensity physical activity can boost our immune system and may help our body to fight off infections such as COVID-19. During this pandemic period, exercise can be much important because it can directly reduce our stress and boost the immune system. The exercise cannot prevent us from getting infected with COVID-19 if exposed. However, staying physically active can boost the immune system and minimize the risk of becoming infected.

\section{Footnotes}

Authors' Contribution: The Whole Work Was Done by Faisal.

Conflict of Interests: The author has no conflict of interest.

Funding/Support: None.

\section{References}

1. World Health Organization (WHO). Stay physically active during self-quarantine. 2020, [cited 2020 May 15]. Available from: http://www.euro.who.int/en/health-topics/health-emergencies/ coronavirus-covid-19/novel-coronavirus-2019-ncov-technicalguidance-OLD/stay-physically-active-during-self-quarantine.

2. Wu C, Chen X, Cai Y, Xia J, Zhou X, Xu S, et al. Risk factors associated with acute respiratory distress syndrome and death in patients with coronavirus disease 2019 pneumonia in Wuhan, China. JAMA Intern Med. 2020. doi: 10.1001/jamainternmed.2020.0994. [PubMed: 32167524]. [PubMed Central: PMC7070509]. 
3. Zhou F, Yu T, Du R, Fan G, Liu Y, Liu Z, et al. Clinical course and risk factors for mortality of adult inpatients with COVID-19 in Wuhan, China: A retrospective cohort study. Lancet. 2020;395(10229):1054-62. doi:10.1016/s0140-6736(20)30566-3.

4. Shaw SCK. Hopelessness, helplessness and resilience: The importance of safeguarding our trainees' mental wellbeing during the COVID-19 pandemic. Nurse Educ Pract. 2020;44:102780. doi: 10.1016/j.nepr.2020.102780. [PubMed: 32272394]. [PubMed Central: PMC7138173].

5. Hilde GN. Exercise and immunity, current issues in sports and exercise medicine, Michael Hamlin, Nick Draper and Yaso Kathiravel, IntechOpen. 2013, [cited 2020 May 31]. Available from: https://www.intechopen.com/books/current-issues-in-sportsand-exercise-medicine/exercise-and-immunity.

6. Simpson RJ, Katsanis E. The immunological case for staying active during the COVID-19 pandemic. Brain Behav Immun. 2020;87:6-7. doi: 10.1016/j.bbi.2020.04.041. [PubMed: 32311497]. [PubMed Central: PMC7165095].

7. Sellami M, Gasmi M, Denham J, Hayes LD, Stratton D, Padulo J, et al. Effects of acute and chronic exercise on immunological parameters in the elderly aged: Can physical activity counteract the effects of aging? Front Immunol. 2018;9:2187. doi: 10.3389/fimmu.2018.02187. [PubMed: 30364079]. [PubMed Central: PMC6191490]. 